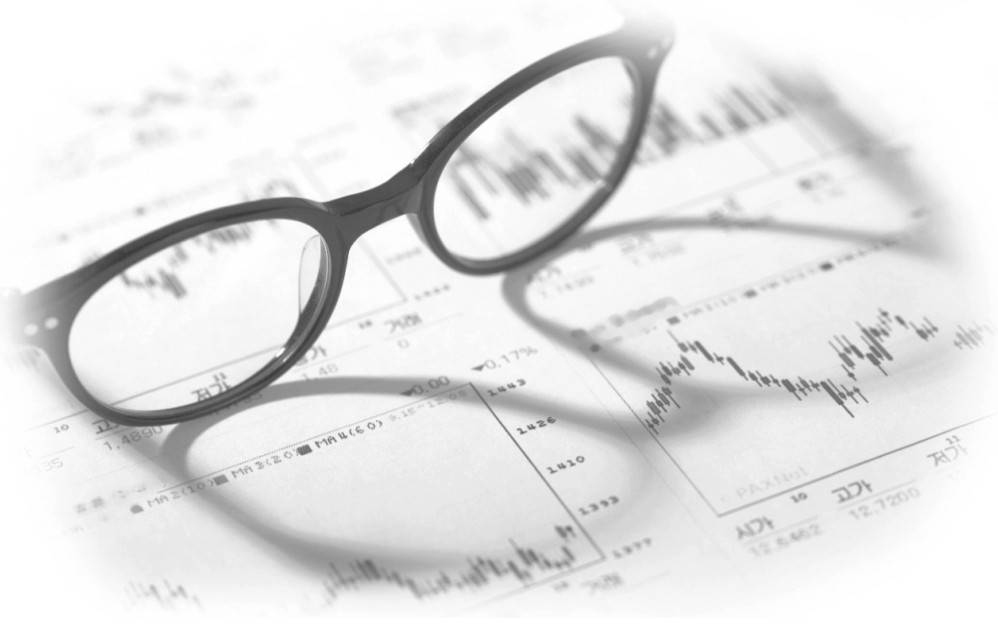

BLOOD RESEARCH

Volume 54 - Number 1 • March 2019

https://doi.org/10.5045/br.2019.54.1.7

Perspective

\section{Cord blood 2.0: state of the art and future directions in transplant medicine}

\author{
John E. Wagner, M.D. \\ Department of Pediatrics, University of Minnesota, \\ Minneapolis, MN, USA
}

Received on January 2, 2019; Accepted on January 3, 2019

October 6, 2018 marks the 30th anniversary of the first cord blood transplantation (CBT). During that time, ans estimated 45,000 CBTs have occurred in adults and children, primarily for the treatment of hematopoietic malignancies but also marrow failure, immunodeficiency, selected metabolic disorders and hemoglobinopathy [1,2]. Fortunately, CBT has addressed some of the most pressing obstacles of allogeneic hematopoietic stem cell (HSC) transplantation generally, namely, rapid access, increased donor safety, less stringent HLA match requirement and potentially lower relapse and chronic graft-versus-host disease (GVHD) risk relative to other HSC sources. But, it too has its challenges, principally, limited cell dose and consequent delays in lympho-hematopoietic recovery.

In the first generation of studies (referred to as CB 1.0), investigators at the University of Minnesota where nearly 2000 CBTs have been performed, identified the CD34 cell dose threshold [3], pioneered the double CBT platform [4-6], and optimized conditioning regimens specifically for CBT, i.e. total body irradiation (TBI) $1320 \mathrm{cGy}$, cyclophosphamide (CY) $120 \mathrm{mg} / \mathrm{kg}$ and fludarabine (FLU) $75 \mathrm{mg} / \mathrm{m}^{2}$ (TCF) [7]. In 2012, we initiated a first in human trial sponsored by Novartis and Magenta Therapeutics to test the safety and efficacy of ex vivo expansion culture to overcome the obstacle of low cell dose using an aryl hydrocarbon antagonist (AHRa) in the presence of stimulatory cytokines. In the initial studies, using the double $\mathrm{CB}$ platform with one unit expanded and the other unit unmodified as an additional safety measure, we showed that 18 of 18 patients engrafted at a median of 14.5 days (range, 7-23 days) after myeloablative conditioning (MAC) with TCF, substantially better than identically-treated historical controls $(P<0.01)$ [8]. Based on these results, 18 additional patients received an expanded $\mathrm{CB}$ product alone, 9 with the same MAC and another 9 treated with non-myelablative conditioning (NMAC) consisting of CY $50 \mathrm{mg} / \mathrm{kg}$, FLU $200 \mathrm{mg} / \mathrm{m}^{2}$ and TBI 200 cGy (Wagner et al., ASH abstract 2017).

Similar to prior reports, expansion culture yielded a median of $1.2 \times 10^{9} \mathrm{CD} 34+$ cells, markedly greater than the input number of $4.2 \times 10^{6}$-a 324-fold (range, 42-1,643 fold) expansion of CD34+ cells. As transplant results vary by intensity of the conditioning regimen, patient outcomes were compared to similarly treated historical cohorts $(\mathrm{N}=151$ MAC; 132 NMAC). For both groups, demographics were similar. For recipients of MAC, CB after expansion culture engrafted in $100 \%$ at a median of 14 days (range, 7-32 days), better than that in the historical cohort transplanted with one or two CB units without expansion where engraftment occurred in $89 \%$ at a median of 23 days (range, 19-31 days) $(P<0.01)$. Complete chimerism was rapid for both $\mathrm{T}$ and myeloid cells with no late graft failures. For recipients of NMAC, the expanded $\mathrm{CB}$ also engrafted in $100 \%$ but at a median of 7 days (range, 6-14 days) as compared to $95 \%$ at a median of 15 days (range, 7-22 days) after unmodified double CBT. In conclusion, the use of an AHRa-based HSC expansion culture accelerates hematopoietic recovery and reduces the risk of graft failure after CBT. In addition, such robust expansion permits us to reconsider the cell dose threshold when selecting the $\mathrm{CB}$

(2) This is an Open Access article distributed under the terms of the Creative Commons Attribution Non-Commercial License (http://creativecommons.org/licenses/by-nc/4.0) 8 which permits unrestricted non-commercial use, distribution, and reproduction in any medium, provided the original work is properly cited. 
graft. Rather than a lower limit of $3.0 \times 10^{7}$ nucleated cell $/ \mathrm{kg}$, future studies will allow a lower limit of $1.0 \times 10^{7}$ nucleated cells $/ \mathrm{kg}$, widening the pool of potential donor units for consideration and therefore, increasing the chance of a better HLA match.

While engraftment has been the principal challenge for CBT, GVHD has been a long standing obstacle for all HSC sources. T cells mediate GVHD which leads to direct tissue damage and the need for prolonged courses of pharmacological immune suppression. Ex vivo T cell depletion is an effective strategy for reducing the risk of GVHD but it is associated with increased risks of graft failure, opportunistic infection and relapse [9], as conventional CD4+ and CD8+ T cells facilitate engraftment, accelerate immune reconstitution, and play a crucial role in the graft-versus-malignancy (GVM) effect of allogeneic HSC. Therefore, we and others have investigated alternative approaches. One strategy has been to isolate a rare population of thymic-derived CD4+CD25+FoxP3+ T cells [referred to as regulatory $\mathrm{T}$ cells (Treg)] found to be critical in maintaining self-tolerance and immune homeostasis [10-14]. In several murine models of allogeneic HSCT across MHC class I and II barriers, lethal GVHD was suppressed when freshly isolated or ex vivo-expanded polyclonal FoxP3+ Treg were co-infused with $\mathrm{T}$ cells. On the basis of these compelling data, we developed a method for isolating and expanding the CD4+CD25+FoxP3+ Treg population in CB that could be used with any HSC source [15]. Crucial for maintaining maternal-fetal tolerance, their high frequency in CB make it an ideal source for adoptive immunotherapy. Following a $18 \pm 1$-day expansion culture incorporating anti-CD3/anti-CD28 antibody-coated beads and IL-2, we initiated a "first-in-human" dose escalation of CB Treg from 0.01 to $3 \times 10^{6} \mathrm{Treg} / \mathrm{kg}$ in double CBT patients [16]. There were no infusional toxicities and GVHD was significantly reduced ( $43 \%$ vs. $61 \% ; P=0.05$ ) with no obvious adverse effect on overall risks of infection and relapse with $>2$ years of follow-up. Based on distinguishing HLA markers that permitted detection of the culture-expanded Treg, Treg were detectable in the PB for up to 14 days (peak on day +2 ). However, greater numbers of Treg were needed to prevent GVHD. We subsequently developed a more robust expansion strategy, using the K562 cell-line that stably expressed CD64 FcR to allow loading with anti-CD3 for $\mathrm{T}$ cell signaling and CD86 for $\mathrm{T}$ cell co-stimulation. In a second dose escalation trial, we infused 3 to $100 \times 10^{6} \mathrm{Treg} / \mathrm{kg}$ (ie, ratio up to $1: 10$ ). Here, we significantly reduced the risk of GVHD from $48 \%$ in Siro/MMF treated recipients to $12 \%(P<0.01)$ [17]. In conclusion, this is the first clear demonstration that ex vivo expanded Treg are potent suppressors of acute GVHD in humans and sets the stage for future clinical trials using CB Treg for prevention of GVHD after PBSCT. While an important HSC source for transplantation, CB may also be an important source of $\mathrm{T}$ cell populations for adoptive immunotherapy.
Today, at the 30th anniversary of CBT, CB holds substantial promise both as a source of HSC and lymphocyte subsets for adoptive cellular therapies. While costs of cell acquisition and longer initial hospitalizations have long been barriers to its wider use, CB is being re-evaluated as more reports demonstrate high survival rates in patients with hematologic malignancy [7], potent GVM in patients with minimal residual disease [18], and rapid engraftment, fewer hospital days and lesser healthcare resource utilization after ex vivo expansion culture by various methods. In the next generation of studies (CB 2.0) we will focus on the broader use of $\mathrm{CB}$ as a source of adoptive cellular therapy to further improve outcomes after HSCT regardless of HSC source and in the treatment of new indications.

\section{Authors' Disclosures of Potential Conflicts of Interest}

Prof. John Wagner is an advisor to Magenta Therapeutics. This relationship has been reviewed and managed by the University of Minnesota in accordance with its conflict of interest policy.

\section{REFERENCES}

1. Ballen KK, Gluckman E, Broxmeyer HE. Umbilical cord blood transplantation: the first 25 years and beyond. Blood 2013;122: 491-8.

2. Smith AR, Wagner JE. Alternative haematopoietic stem cell sources for transplantation: place of umbilical cord blood. Br J Haematol 2009;147:246-61.

3. Wagner JE, Barker JN, DeFor TE, et al. Transplantation of unrelated donor umbilical cord blood in 102 patients with malignant and nonmalignant diseases: influence of CD34 cell dose and HLA disparity on treatment-related mortality and survival. Blood 2002;100:1611-8.

4. Barker JN, Weisdorf DJ, Wagner JE. Creation of a double chimera after the transplantation of umbilical-cord blood from two partially matched unrelated donors. N Engl J Med 2001;344: 1870-1.

5. Barker JN, Weisdorf DJ, DeFor TE, et al. Transplantation of 2 partially HLA-matched umbilical cord blood units to enhance engraftment in adults with hematologic malignancy. Blood 2005;105:1343-7.

6. Brunstein CG, Barker JN, Weisdorf DJ, et al. Umbilical cord blood transplantation after nonmyeloablative conditioning: impact on transplantation outcomes in 110 adults with hematologic disease. Blood 2007;110:3064-70.

7. Eapen M, Kurtzberg J, Zhang MJ, et al. Umbilical cord blood transplantation in children with acute leukemia: impact of conditioning on transplantation outcomes. Biol Blood Marrow Transplant 2017;23:1714-21.

8. Wagner JE Jr, Brunstein CG, Boitano AE, et al. Phase I/II trial of stemregenin-1 expanded umbilical cord blood hematopoietic stem cells supports testing as a stand-alone graft. Cell Stem Cell 2016;18:144-55.

9. Wagner JE, Thompson JS, Carter SL, Kernan NA; Unrelated 
Donor Marrow Transplantation Trial. Effect of graft-versus-host disease prophylaxis on 3-year disease-free survival in recipients of unrelated donor bone marrow (T-cell Depletion Trial): a multi-centre, randomised phase II-III trial. Lancet 2005;366: 733-41.

10. Cohen JL, Trenado A, Vasey D, Klatzmann D, Salomon BL. CD4(+)CD25(+) immunoregulatory T cells: new therapeutics for graft-versus-host disease. J Exp Med 2002;196:401-6.

11. Hoffmann P, Ermann J, Edinger M, Fathman CG, Strober S. Donor-type CD4(+)CD25(+) regulatory T cells suppress lethal acute graft-versus-host disease after allogeneic bone marrow transplantation. J Exp Med 2002;196:389-99.

12. Taylor PA, Noelle RJ, Blazar BR. CD4(+)CD25(+) immune regulatory cells are required for induction of tolerance to alloantigen via costimulatory blockade. J Exp Med 2001;193: 1311-8.

13. Taylor PA, Lees CJ, Blazar BR. The infusion of ex vivo activated and expanded CD4(+)CD25(+) immune regulatory cells inhibits graft-versus-host disease lethality. Blood 2002;99:3493-9.
14. Taylor PA, Panoskaltsis-Mortari A, Swedin JM, et al. L-Selectin(hi) but not the L-selectin(lo) CD4+25+ T-regulatory cells are potent inhibitors of GVHD and BM graft rejection. Blood 2004;104:3804-12.

15. McKenna DH Jr, Sumstad D, Kadidlo DM, et al. Optimization of cGMP purification and expansion of umbilical cord bloodderived T-regulatory cells in support of first-in-human clinical trials. Cytotherapy 2017;19:250-62.

16. Brunstein CG, Miller JS, Cao Q et al. Infusion of ex vivo expanded $\mathrm{T}$ regulatory cells in adults transplanted with umbilical cord blood: safety profile and detection kinetics. Blood 2011;117: 1061-70.

17. Brunstein CG, Miller JS, McKenna DH, et al. Umbilical cord blood-derived T regulatory cells to prevent GVHD: kinetics, toxicity profile, and clinical effect. Blood 2016;127:1044-51.

18. Milano F, Gooley T, Wood B, et al. Cord-blood transplantation in patients with minimal residual disease. N Engl J Med 2016; 375:944-53. 